\title{
Flora of inland Atlantic riparian forests in southwestern Brazil
}

\author{
Claudenice Faxina $^{1,3}$, Erich Fischer ${ }^{2}$ \& Arnildo Pott $^{1}$ \\ ${ }^{1}$ Universidade Federal do Mato Grosso do Sul, Programa de Pós-Graduação em Biologia Vegetal, \\ Campo Grande, MS, Brazil. \\ ${ }^{2}$ Universidade Federal do Mato Grosso do Sul, Centro de Ciências Biológicas e da Saúde, \\ Campo Grande, MS, Brazil. \\ ${ }^{3}$ Corresponding author: Claudenice Faxina, e-mail: clau.zucca@gmail.com; clau.zucca@gebio.org.br
}

FAXINA, C., FISCHER, E., POTT, A. Flora of inland Atlantic riparian forests in southwestern Brazil Biota Neotropica. 15(3): e20130083. http://dx.doi.org/10.1590/1676-06032015008313

\begin{abstract}
The flora of the Atlantic Rainforest of Mato Grosso do Sul, southwestern Brazil, has not been inventoried in spite of being the westernmost inland region of this domain. We present an inventory of the riparian flora of inland Atlantic Forest in Mato Grosso do Sul. We describe the species distribution along three habitats with contrasting flood conditions: non-flooded, seasonally flooded, and swampy forests. The inventory consisted of sampling every reproductive individual, during 12 months on 1.12 ha of plots inside a 24 ha study area. We recorded 1967 individuals of 253 species and 72 families. The most representative families in number of species were Asteraceae (27), Fabaceae (19), Myrtaceae (17), Cyperaceae (12), Rubiaceae, Solanaceae and Orchidaceae (10 each). The most abundant reproductive species were Guarea macrophylla (169 individuals), Miconia chamissois (85) and Conyza bonariensis (80). Eleven species of six families were recorded for the first time in Mato Grosso do Sul, two of them endemic to the Atlantic Forest - Passiflora jilekii and Capanema micromera. We found 119 species exclusively in non-flooded habitat, 19 in seasonally flooded habitat, and 31 in swampy forest. Guarea macrophylla was the most frequent species in swampy forest, and Gochnatia polymorpha in non-flooded. The riparian forest flora at the study site resembles the Atlantic Forest and includes wide distribution riparian species; the variation of flood conditions among habitats favors its richness. Our records add new occurrences for Mato Grosso do Sul and new distribution ranges for some plant species, what arises concern upon the local biodiversity conservation.
\end{abstract}

Keywords: flood gradient, plant distribution, plant richness, riparian forest, semideciduous forest.

FAXINA, C., FISCHER, E., POTT, A. Flora de florestas ripárias da Mata Atlântica de interior no sudoeste do Brasil. Biota Neotropica. 15(3): e20130083. http://dx.doi.org/10.1590/1676-06032015008313

Resumo: Vegetação ripária de Floresta Atlântica de interior no sudoeste do Brasil. A flora da Mata Atlântica de Mato Grosso do Sul, sudoeste do Brasil, tem sido muito pouco estudada, embora represente a porção mais oeste e continental deste domínio. Descrevemos aqui a flora de matas ciliares na região de Mata Atlântica de Mato Grosso do Sul, e a distribuição das espécies em três habitas classificados segundo a ocorrência de inundação como: floresta não inundável, floresta sazonalmente inundável, e floresta inundada. O inventário consistiu de um ano de amostragens mensais de todos os indivíduos reprodutivos em 1,12 ha de parcelas em 24 ha. Encontramos 1967 indivíduos de 253 espécies e 72 famílias. As famílias mais representativas foram Asteraceae (27), Fabaceae (19), Myrtaceae (17), Cyperaceae (12), Rubiaceae, Solanaceae e Orchidaceae (10 espécies cada). As espécies reprodutivas mais abundantes foram Guarea macrophylla (169), Miconia chamissois (85) e Conyza bonariensis (80). Onze espécies de seis famílias foram registradas pela primeira vez em Mato Grosso do Sul, duas delas endêmicas da Mata Atlântica - Passiflora jilekii e Capanema micromera. Encontramos 119 espécies apenas na floresta não inundável, 19 na floresta sazonalmente inundável, e 31 na floresta inundada. Guarea macrophylla foi a espécie mais comum na floresta inundada e Gochnatia polymorpha, na floresta não inundável. A flora das matas ciliares estudadas assemelha-se à da Mata Attântica e apresenta espécies ripárias de ampla distribuição; a variação do regime de inundação entre os habitats favorece sua riqueza. Nossos registros adicionam novas ocorrências para Mato Grosso do Sul e novos limites de distribuição para algumas espécies, fatos que trazem preocupação quanto à conservação da biodiversidade local.

Palavras-chave: distribuição de plantas, gradiente de inundação, floresta ripária, floresta semidecídua, riqueza de espécies. 


\section{Introduction}

Floristic inventories are important to know plant species distribution and diversity, and to subsidize conservation initiatives. The southeastern Mato Grosso do Sul's territory has been considered part of the Atlantic Forest domain, indeed its westernmost inland limit (Rizzini 1979). However, this region is widely uncovered regarding plant surveys. Most inventory efforts in the state of Mato Grosso do Sul have focused on the Pantanal and its surrounding Cerrado floras (Pott \& Pott 1994, 2000; 2003, Salis et al. 2004, Pott et al. 2006, Lehn et al. 2008, Damasceno-Junior et al. 2009, Noguchi et al. 2009). Additional plant surveys are available for the northeastern and southwestern Cerrado regions of the state (Pott et al. 2006, Arruda \& Daniel 2007, Baptista-Maria et al. 2009). This situation occurs, in part, because the Atlantic Forest domain in Mato Grosso do Sul was largely modified for agriculture, and the remaining natural vegetation became limited to riparian forests.

Riparian forests are highly diverse, often presenting low similarity of flora even between adjacent portions (Rodrigues \& Nave 2004). Variation of flood conditions is largely associated with heterogeneity in floristic composition and life forms along riparian forests (Fischer \& Araujo 1995, Rodrigues \& Shepherd 2004). The importance of abiotic and biotic pressures appears to vary along the gradient of flooding. Increased flood depth and duration can select plant taxa whose traits allow escaping from waterlogging, whereas competitive ability is expected to determine plant occurrences in non-flooded forest patches (Fischer \& Santos 2001, Rodrigues \& Shepherd 2004). Here, we describe the flora of riparian forests along Touro and Tarumã streams in the Mato Grosso do Sul's Atlantic Forest domain, and address species distribution among three contiguous habitats with contrasting flood conditions.

\section{Methods}

\section{Study site}

The study was carried out in five plots within 24 ha of the Touro and Tarumã riparian vegetation in the Paraná river basin, municipality of Naviraí, Mato Grosso do Sul (Figure 1). The climate is seasonal characterized by rainy summers and dry winters (Cfa of Köppen); mean annual temperature varies from 12 to $28^{\circ} \mathrm{C}$ and mean annual rainfall is $1600 \mathrm{~mm}$ (IBGE 1984). The region belongs to the Caiuá geological formation, from upper Cretaceous, with $360 \mathrm{~m}$ average altitude and fertile soils, mainly Red Latosol and small patches of Red Spodosol (IBGE 1984), and hydromorphic soils on floodable or swampy areas. The study site is located in the western portion of the major continental phytogeographic domain of Atlantic Rainforest (Ab'Saber 2000). Remaining vegetation comprises mainly alluvial or submontane semideciduous forests (Veloso et al. 1991). Hereafter we refer to the study alluvial semideciduous forests simply as 'riparian forests'.
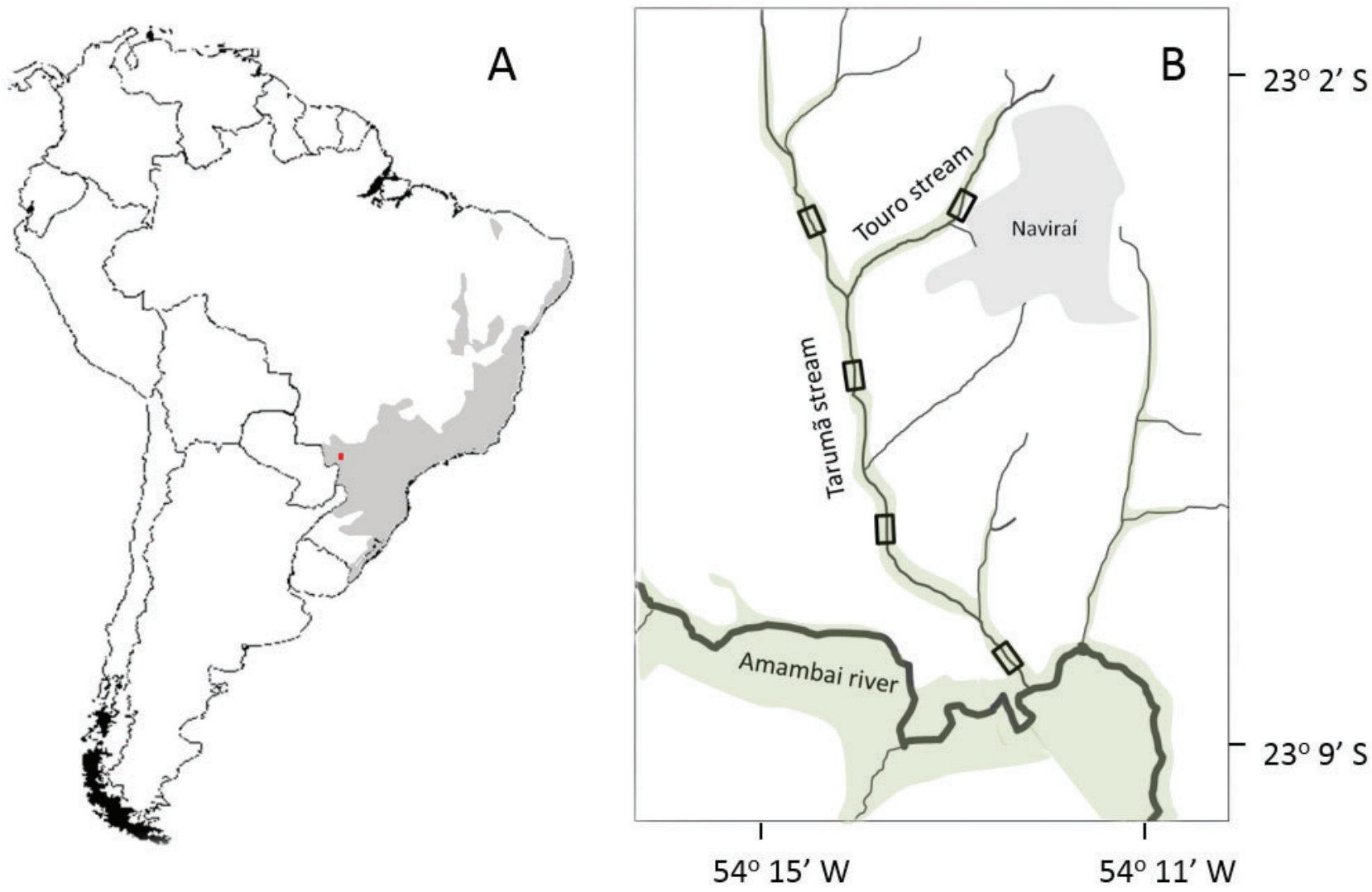

Figure 1. (A) Atlantic Forest domain (gray) and the study site (red dot). (B) Location of plant surveys (rectangles) in the Touro and Tarumã riparian forests (greenish); gray indicates the urban area of Naviraí, and white does pastures and cultivated areas.

Figura 1. (A) Domínio da Mata Atlântica (cinza) e local de estudo (ponto vermelho). (B) Localização das amostragens de plantas (retângulos) nas florestas ripárias (esverdeado) do Touro e Tarumã; cinza indica a área urbana de Naviraí, e branco indica pastagens e áreas cultivadas. 


\section{Data collection and analysis}

From July 2009 to June 2010 we monthly surveyed all reproductive plant individuals in six meters wide plots perpendicular to the streams, to include the flood gradient from the river margin to the higher ground of the riparian forest. Plots were placed at least $30 \mathrm{~m}$ apart, and their lengths varied from 40 to $180 \mathrm{~m}$ according to the riparian forest width at each stretch. The total sampled area was 1.12 ha. To control previously sampled individuals, we marked them with numbered aluminum tags. Samples included all angiosperms of different habits (Guedes-Bruni et al. 2002): ground herbs (without woody stems above ground), shrubs (multiple woody stems), climbers (woody or non-woody with climbing structures), trees (one woody stem; palms included), epiphytes, and parasites. For some species of shrubs and herbs in tussocks or multi-stemmed clumps, each bunch was counted as one individual. We also recorded monthly the flood condition (flooded or not flooded) where individuals, or their hosts if epiphyte or parasite, were rooted. After one year of records of flood conditions we assumed three habitat categories: (1) soil not flooded (nonflooded habitat, hereafter), (2) soil flooded during the rainy season (seasonally flooded habitat), and (3) soil persistently flooded (swampy habitat). We then determined limits of habitats within the plots and estimated the area covered by them with help of field GPS readings checked on Google Earth images, where we measured distances between zones. The altimetric difference between the lowest and the upper sampled areas was ca $43 \mathrm{~m}$. Based on the surveyed area for each habitat (nonflooded $=0.64$ ha, seasonally flooded $=0.22$ ha, and swampy $=0.26 \mathrm{ha}$ ) we calculated density of reproductive individuals per species $\left(\mathrm{N}^{-h^{-1}}{ }^{-1}\right)$ per habitat. We then used values of density per habitat as a proxy for number of individuals after controlling for equal size of areas ( $1 \mathrm{ha}$ ) among habitats. Considering the total of individuals estimated for one hectare of each habitat, we calculated the proportion ( $\%$ ) of individuals per habitat for each species separately. Finally we entered the values of proportion of individuals in a species $\mathrm{x}$ habitat matrix to ordinate plant species in relation to habitats, in the $\mathrm{R}$ program ( $\mathrm{R}$ Development Core Team 2010); epiphytes and species with less than 10 individuals were not included. Number of surveyed species relative to sample size was evaluated through rarefaction curves; in the Past program (Hammer et al. 2001). Identifications were based on comparisons with herbarium materials, and help of literature and specialists. Taxonomy follows the Angiosperm Phylogeny Group (APG III 2009). Voucher material was included in the Herbarium CGMS, Universidade Federal de Mato Grosso do Sul. Finally, we obtained from literature and herbarium sites (www.tropicos.org; www.floradobrasil.jbrj.gov.br) the occurrences of species throughout the phytogeographical domains. Each species was assigned to a domain when its previous known occurrence was restricted, or assigned as "wide distribution" when its occurrence included more domains, considered as Amazonia, Atlantic Forest, Caatinga, Cerrado, Chaco, Pampas or Pantanal. Species that are not South American natives were assigned as exotic.

\section{Results}

Surveyed flora and new occurrences in Mato Grosso do Sul

We recorded 1967 reproductive individuals of 253 species, 193 genera and 72 families (Table 1). The eight richest families included $46 \%$ of all surveyed species - Asteraceae (27 species), Fabaceae (19), Myrtaceae (17), Cyperaceae (12), Malvaceae, Orchidaceae, Rubiaceae and Solanaceae (10). Thirty-three families presented two to nine species, and the remaining families presented a single one. The most abundant reproductive species were Guarea macrophylla (Meliaceae; 169 individuals), Miconia chamissois (Melastomataceae; 85), Conyza bonariensis (Asteraceae; 80), Ocotea lancifolia (Lauraceae; 65) and Cecropia pachystachya (Urticaceae; 57). Eleven species were recorded for the first time in Mato Grosso do Sul - Campylocentrum grisebachii, Capanemia micromera, Cohniella jonesiana, Microlaelia lundii, Rodriguezia decora, Sauroglossum nitidum (all Orchidaceae), Passiflora jilekii (Passifloraceae), Persea willdenovii (Lauraceae), Merremia macrocalyx (Convolvulaceae), Qualea cordata (Vochysiaceae), and the saprophyte Apteria aphylla (Burmanniaceae). We additionally recorded the parasite Phoradendron piperoides (Viscaceae) and four invader species - Leucaena leucocephala (Fabaceae), Tecoma stans (Bignoniaceae), Cordia myxa (Cordiaceae), and Hedychium coronarium (Zingiberaceae). Most species were herbs $(99 ; 39 \%)$ or trees $(72 ; 28 \%)$. Climbers and shrubs included $39(15 \%)$ and $33(13 \%)$ species, respectively, and epiphytes, nine species (4\%).

\section{Species distribution among habitats}

Floras of swampy and seasonally flooded forests showed less species and so tended to be fully surveyed, but flora of nonflooded forest was richer and less completely surveyed despite its larger area sampled (Figure 2). In general, most species (169 out of 253) were sampled only in one habitat, mainly in nonflooded (119) than in swampy (31) or seasonally flooded (19) (Table 1). On the other hand, considering only those species with 10 or more individuals recorded ( $n=49$ species), $63 \%$ were found in the three habitats, $20 \%$ in two habitats, $13 \%$ exclusively in non-flooded, $4 \%$ in swampy, and none was found solely in seasonally flooded habitat. In overall, densities of the most abundant herbs and shrubs species in swampy and seasonally flooded habitats were one order of magnitude above non-flooded habitat. Among tree species, densities in swampy were one order of magnitude above the other habitats.

Among herbaceous plants, Conyza bonariensis $\left(62.5\right.$ N.ha $\left.{ }^{-1}\right)$, Geophila repens (57.8) and Triumfetta bartramia (45.3) were the species with highest densities in non-flooded habitats; G. repens, Elephantopus mollis (32.8) and Rhynchospora marisculus (23.4) occurred in this habitat only (Table 1). The commonest species in swampy habitat were Coccocypselum lanceolatum (153.9) and Calyptrocarya glomerulata (119.2), whereas Conyza bonariensis (182.6) and Canna indica (160.0) were the most common in seasonally flooded habitat. Among shrubs, highest densities in non-flooded habitat were found for Piper aduncum (32.8), Psychotria carthagenensis (31.3) and Ludwigia sericea (23.4), and in swampy for Miconia chamissois (28.8), M. theizans (13.9) and Gaylussacia brasiliensis (61.5) (Table 1). Density of Piper aduncum (122.7) was one order of magnitude higher than the subsequent most abundant species in seasonally flooded habitat, M. chamissois (45.5), Lantana camara (31.8) and Ludwigia sericea (22.7). The commonest tree species in non-flooded habitat were Gochnatia polymorpha (34.4), Allophylus edulis (31.25) and Helietta apiculata (28.1); G. polymorpha and $H$. apiculata were exclusively found in this habitat (Table 1). Guarea macrophylla (557.7), Ocotea lancifolia (188.5) and Styrax 
Faxina, C. et al.

Table 1. Families, species, habits $(\mathrm{TRE}=$ trees; SHR = shrubs; EPI $=$ epiphytes; HER = herbs; CLI = climbers; PAR = parasites), number of individuals $(\mathrm{n})$, and density $\left(\mathrm{N} . \mathrm{ha}^{-1}\right)$ in three soil moisture types $(\mathrm{NF}=$ non flooded; $\mathrm{SF}=$ seasonally flooded; $\mathrm{SW}=$ swampy) in the riparian forests along Touro and Tarumã streams, Paraná river basin, Mato Grosso do Sul. Collection number: $\mathrm{CF}=$ Claudenice Faxina; Phytogeography: $\mathrm{AF}=$ Atlantic Forest, $\mathrm{CE}=$ Cerrado, $\mathrm{CH}=$ Chaco, $\mathrm{WD}=$ wide distribution, $\mathrm{EX}=$ exotic.

Tabela 1. Famílias, espécies, hábitos (TRE = árvores; SHR = arbustos; EPI=epífitas; HER = herbáceas; CLI = climbers; PAR= parasita), número de indivíduos (n) e densidade (N.ha-1) em três tipos umidade de solo (NF = não inundado; $\mathrm{SF}=$ sazonalmente inundado; $\mathrm{SW}=$ permanentemente inundado) ao longo da floresta ripária dos córregos Touro e Tarumã, bacia do rio Paraná, Mato Grosso do Sul. Número de coleta: $\mathrm{CF}=$ Claudenice Faxina; Fitogeografia: $\mathrm{AF}=$ Mata Atlântica, $\mathrm{CE}=$ Cerrado, $\mathrm{CH}=$ Chaco, $\mathrm{WD}=$ ampla distribuição, $\mathrm{EX}=$ exótica.

\begin{tabular}{|c|c|c|c|c|c|c|c|c|}
\hline Family & Species & Habit & $\mathbf{n}$ & NF & SF & SW & $\begin{array}{l}\text { Phyto- } \\
\text { geography }\end{array}$ & $\begin{array}{c}\text { Collection } \\
\text { number }\end{array}$ \\
\hline \multirow[t]{2}{*}{ Acanthaceae } & Hygrophila costata Nees & HER & 1 & 1.6 & & & WD & \\
\hline & Ruellia $\mathrm{cf}$. brevifolia (Pohl) C. Ezcurra & SHR & 1 & 1.6 & & & WD & CF 075 \\
\hline \multirow[t]{2}{*}{ Alismataceae } & Echinodorus longipetalus Micheli & HER & 4 & & & 15.4 & WD & CF 229 \\
\hline & Sagittaria rhombifolia Cham. & HER & 1 & & & 3.9 & WD & \\
\hline \multirow[t]{2}{*}{ Anacardiaceae } & Schinus terebenthifolia Raddi & TRE & 3 & 4.7 & & & WD & CF 042 \\
\hline & Tapirira guianensis Aubl. & TRE & 32 & 7.8 & 36.4 & 73.8 & WD & \\
\hline \multirow[t]{3}{*}{ Apocynaceae } & Condylocarpon isthmicum (Vell.) A. DC. & CLI & 7 & 3.2 & 4.6 & 15.4 & $\mathrm{AF}$ & CF 238 \\
\hline & Mesechites sp. & CLI & 1 & 1.6 & & & WD & CF 217 \\
\hline & Tabernaemontana catharinensis A. DC. & SHR & 1 & 1.6 & & & $\mathrm{AF}$ & \\
\hline \multirow[t]{2}{*}{ Aquifoliaceae } & Ilex brasiliensis Loes. & TRE & 13 & 1.6 & 9.1 & 38.5 & $\mathrm{AF}$ & CF 228 \\
\hline & Ilex affinis Gardner & TRE & 2 & & & 7.7 & WD & CF 174 \\
\hline \multirow[t]{2}{*}{ Araliaceae } & Hydrocotyle leucocephala Cham. \& Schltdl. & HER & 9 & 1.6 & 4.6 & 27.9 & WD & \\
\hline & Hydrocotyle verticillata Thunb. & HER & 1 & & 4.6 & & WD & \\
\hline \multirow[t]{3}{*}{ Arecaceae } & Acrocomia aculeata (Jacq.) Lodd. ex Mart. & TRE & 5 & 7.8 & & & WD & \\
\hline & Geonoma brevispatha Barb. Rodr. & TRE & 12 & & & 46.2 & $\mathrm{AF}$ & CF 011 \\
\hline & Syagrus romanzoffiana (Cham.) Glassman & TRE & 14 & 9.4 & 18.2 & 15.4 & $\mathrm{AF}$ & \\
\hline Aristolochiaceae & Aristolochia triangularis Cham. & CLI & 3 & 4.7 & & & $\mathrm{AF}$ & CF 081 \\
\hline \multirow[t]{27}{*}{ Asteraceae } & Achyrocline satureioides (Lam.) DC. & HER & 5 & 7.8 & & & WD & \\
\hline & Aspilia latissima Malme & HER & 2 & 1.6 & 3.9 & & WD & \\
\hline & Baccharis sp. & HER & 1 & 1.6 & & & & CF 302 \\
\hline & $\begin{array}{l}\text { Barrosoa candolleana (Hook. \& Arn.) R. M. } \\
\text { King \& H. Rob. }\end{array}$ & HER & 1 & & & 3.9 & WD & CF 274 \\
\hline & Conyza bonariensis (L.) Cronquist & HER & 80 & 62.5 & 182.6 & & WD & CF 262 \\
\hline & $\begin{array}{l}\text { Chromolaena odorata (L.) R. M. King \& H. } \\
\text { Rob. }\end{array}$ & HER & 11 & 9.4 & 18.2 & 3.9 & WD & CF 063 \\
\hline & Eclipta prostrata (L.) L. & HER & 2 & & & 7.7 & WD & CF 337 \\
\hline & Elephantopus mollis Kunth & HER & 21 & 32.8 & & & WD & CF 125 \\
\hline & Elephantopus palustris Gardner & HER & 2 & 1.6 & 3.9 & & WD & CF 112 \\
\hline & Emilia fosbergii Nicolson & HER & 2 & 3.1 & & & WD & CF 141 \\
\hline & Erechtites hieraciifolius (L.) Raf. ex DC. & HER & 2 & & 10.0 & & WD & CF 183 \\
\hline & Gnaphalium pensylvanicum Willd. & HER & 1 & & 4.6 & & WD & CF 143 \\
\hline & Gochnatia polymorpha (Less.) Cabrera & SHR & 22 & 34.4 & & & WD & CF 044 \\
\hline & $\begin{array}{l}\text { Gymnocoronis spilanthoides (D. Don ex Hook. } \\
\text { \& Arn.) DC. }\end{array}$ & HER & 1 & & & 3.9 & WD & CF 118 \\
\hline & Mikania cordifolia (L. f.) Willd. & CLI & 2 & 1.6 & 4.6 & & WD & CF 041 \\
\hline & Mikania micrantha Kunth & CLI & 6 & 9.4 & & & WD & CF 335 \\
\hline & Porophyllum ruderale (Jacq.) Cass. & HER & 3 & 4.7 & & & WD & CF 209 \\
\hline & Sonchus oleraceus L. & HER & 2 & 3.1 & & & $\mathrm{EX}$ & CF 128 \\
\hline & Trichogonia crenulata (Gardner) D. J. N. Hind & SHR & 2 & & & 7.7 & WD & CF 108 \\
\hline & Trixis antimenorrhoea (Schrank) Kuntze & CLI & 3 & 4.7 & & & WD & CF 155 \\
\hline & Vernonanthura cuneifolia (Gardner) H. Rob. & HER & 6 & & & & WD & CF 139 \\
\hline & Vernonia cf. grandiflora Less. & HER & 2 & 3.1 & & & WD & CF 297 \\
\hline & Vernonia remotiflora Rich. & HER & 4 & 4.7 & 4.6 & & WD & CF 207 \\
\hline & Vernonia sp.1 & HER & 1 & 1.6 & & & & CF 345 \\
\hline & Vernonia sp. 2 & SHR & 5 & 6.3 & 4.6 & & & CF 065 \\
\hline & Indet. 1 & HER & 1 & 1.6 & & & & CF 263 \\
\hline & Indet. 2 & SHR & 1 & 1.6 & & & & CF 338 \\
\hline Begoniaceae & Begonia cucullata Willd. & HER & 22 & 1.6 & 18.2 & 65.4 & WD & CF 216 \\
\hline
\end{tabular}


Table 1. Continued.

\begin{tabular}{|c|c|c|c|c|c|c|c|c|}
\hline Family & Species & Habit & $\mathbf{n}$ & NF & SF & SW & $\begin{array}{l}\text { Phyto- } \\
\text { geography }\end{array}$ & $\begin{array}{l}\text { Collection } \\
\text { number }\end{array}$ \\
\hline \multirow[t]{5}{*}{ Bignoniaceae } & $\begin{array}{l}\text { Anemopaegma chamberlaynii (Sims) Bureau \& } \\
\text { K. Schum. }\end{array}$ & CLI & 1 & 1.6 & & & WD & CF 184 \\
\hline & Fridericia florida (DC.) L.G. Lohmann & CLI & 3 & 4.7 & & & WD & CF 085 \\
\hline & Fridericia $\mathrm{sp}$ & CLI & 1 & 1.6 & & & & CF 145 \\
\hline & Pyrostegia venusta (Ker Gawl.) Miers & CLI & 2 & & 9.1 & & $\mathrm{AF}$ & CF 043 \\
\hline & Tecoma stans (L.) Juss. ex Kunth & SHR & 3 & 4.7 & & & EX & CF 181 \\
\hline \multirow[t]{2}{*}{ Bromeliaceae } & Aechmea bromeliifolia (Rudge) Baker & EPI & 2 & 1.6 & & 3.9 & WD & \\
\hline & Bromelia balansae $\mathrm{Mez}$ & HER & 7 & 4.7 & 13.6 & 3.9 & WD & \\
\hline Burmaniaceae & Apteria aphylla (Nutt.) Barnhart ex Small & HER & 4 & & & 15.4 & WD & CF 199 \\
\hline \multirow[t]{2}{*}{ Boraginaceae } & Cordia myxa L. & TRE & 2 & 1.6 & 4.6 & & $\mathrm{EX}$ & CF 233 \\
\hline & Cordia polycephala (Lam.) I. M. Johnst. & SHR & 5 & 3.1 & 9.1 & 3.9 & WD & CF 248 \\
\hline Cactaceae & Epiphyllum phyllanthus (L.) Haw. & EPI & 1 & 1.6 & & & WD & \\
\hline Campanulaceae & Pratia cf. hederacea (Cham.) G. Don & HER & 2 & & & & WD & \\
\hline Cannaceae & Canna indica $\mathrm{L}$. & HER & 37 & 1.6 & 160.0 & 3.9 & WD & CF 048 \\
\hline Cannabaceae & Trema micrantha (L.) Blume & TRE & 3 & 4.7 & & & WD & CF 130 \\
\hline Cardiopteridaceae & Citronella gongonha (Mart.) R. A. Howard & TRE & 5 & 1.6 & & 15.4 & WD & CF 023 \\
\hline \multirow[t]{2}{*}{ Commelinaceae } & Commelina erecta $\mathrm{L}$. & HER & 2 & & 10.0 & & WD & CF 121 \\
\hline & Floscopa glabrata (Kunth) Hassk. & HER & 2 & 1.6 & 4.6 & & WD & CF 313 \\
\hline \multirow[t]{3}{*}{ Convolvulaceae } & Ipomoea $\mathrm{sp}$. & CLI & 1 & 1.6 & & & & CF 298 \\
\hline & Jacquemontia $\mathrm{sp}$. & CLI & 1 & 1.6 & & & & CF 187 \\
\hline & Merremia macrocalyx (Ruiz \& Pav.) O’Donell & CLI & 1 & 1.6 & & & WD & CF 336 \\
\hline Costaceae & Costus arabicus $\mathrm{L}$. & HER & 11 & & 45.5 & 3.8 & WD & \\
\hline Cucurbitaceae & Melothria cf. pendula L. & CLI & 1 & 1.6 & & & WD & CF 235 \\
\hline \multirow[t]{12}{*}{ Cyperaceae } & Calyptrocarya glomerulata (Brongn.) Urb. & HER & 37 & 3.1 & 18.2 & 119.2 & WD & CF 324 \\
\hline & Cyperus haspan $\mathrm{L}$. & HER & 20 & & 10.0 & 69.2 & WD & CF 311 \\
\hline & Cyperus luzulae (L.) Rottb. ex Retz. & HER & 9 & & 27.3 & 11.5 & WD & CF 260 \\
\hline & Cyperus odoratus L. & HER & 5 & & & 19.2 & WD & CF 256 \\
\hline & Diplacrum longifolium (Griseb.) C. B. Clarke & HER & 6 & & 10.0 & 15.4 & WD & CF 325 \\
\hline & Fimbristylis complanata (Retz.) Link & HER & 3 & & 13.6 & & WD & CF 258 \\
\hline & Fuirena umbellata Rottb. & HER & 4 & & 13.6 & 3.8 & WD & CF 294 \\
\hline & Pycreus lanceolatus (Poir.) C. B. Clarke & HER & 8 & 3.1 & 10.0 & 15.4 & WD & CF 242 \\
\hline & Rhynchospora corymbosa (L.) Britton & HER & 8 & 1.6 & 13.6 & 15.4 & WD & CF 210 \\
\hline & Rhynchospora marisculus Nees & HER & 15 & 23.4 & & & WD & CF 244 \\
\hline & Scleria cf. bancana Miq. & HER & 21 & 7.8 & 22.7 & 42.4 & WD & CF 124 \\
\hline & Scleria melaleuca $\mathrm{Rchb}$. & HER & 26 & 3.1 & 18.2 & 76.9 & WD & \\
\hline \multirow[t]{4}{*}{ Dioscoreaceae } & Dioscorea altissima Lam. & CLI & 4 & 6.3 & & & WD & CF 347 \\
\hline & Dioscorea cf. trifida L. f. & CLI & 1 & & 4.6 & & WD & CF 291 \\
\hline & Dioscorea sp.1 & CLI & 5 & 1.6 & & 15.4 & & CF 096 \\
\hline & Dioscorea $\mathrm{sp} .2$ & CLI & 4 & 1.6 & & 11.5 & & CF 259 \\
\hline Ebenaceae & Diospyros inconstans Jacq. & TRE & 1 & 1.6 & & & WD & CF 059 \\
\hline Ericaceae & Gaylussacia brasiliensis (Spreng.) Meisn. & SHR & 16 & & & 61.5 & WD & CF 107 \\
\hline Eriocaulaceae & Syngonanthus caulescens (Poir.) Ruhland & HER & 4 & & 4.6 & 11.5 & WD & CF 243 \\
\hline \multirow[t]{8}{*}{ Euphorbiaceae } & Acalypha sp. & HER & 1 & & & 3.9 & & \\
\hline & Croton lobatus $\mathrm{L}$. & HER & 1 & 1.6 & & & WD & CF 226 \\
\hline & Croton urucurana Baill. & TRE & 6 & 9.4 & & & WD & CF 060 \\
\hline & Euphorbia heterophylla $\mathrm{L}$. & HER & 1 & 1.6 & & & WD & CF 142 \\
\hline & Ricinus communis L. & TRE & 5 & 7.8 & & & EX & \\
\hline & Sapium haematospermum Müll. Arg. & TRE & 8 & 4.7 & 18.2 & 3.9 & WD & CF 208 \\
\hline & Sebastiania brasiliensis Spreng. & TRE & 18 & 18.8 & 18.2 & 7.7 & WD & CF 055 \\
\hline & Tragia $\mathrm{sp}$. & CLI & 1 & 1.6 & & & & CF 158 \\
\hline \multirow[t]{5}{*}{ Fabaceae } & Canavalia mattogrossensis (Barb. Rodr.) Malme & CLI & 3 & 4.7 & & & WD & CF 319 \\
\hline & $\begin{array}{l}\text { Centrosema sagittatum (Humb. \& Bonpl. ex } \\
\text { Willd.) Brandegee }\end{array}$ & CLI & 2 & 3.1 & & & WD & CF 320 \\
\hline & Chamaecrista nictitans (L.) Moench & HER & 1 & 1.6 & & & WD & CF 343 \\
\hline & Copaifera langsdorffii Desf. & TRE & 2 & 3.1 & & & WD & CF 193 \\
\hline & Crotalaria pallida Aiton & HER & 5 & 7.8 & & & WD & CF 342 \\
\hline
\end{tabular}


Table 1. Continued.

\begin{tabular}{|c|c|c|c|c|c|c|c|c|}
\hline Family & Species & Habit & $\mathbf{n}$ & NF & SF & SW & $\begin{array}{l}\text { Phyto- } \\
\text { geography }\end{array}$ & $\begin{array}{l}\text { Collection } \\
\text { number }\end{array}$ \\
\hline & Desmodium cajanifolium (Kunth) DC. & HER & 1 & & 4.6 & & WD & \\
\hline & Desmodium incanum DC. & HER & 3 & 4.7 & & & WD & CF 299 \\
\hline & Galactia striata (Jacq.) Urb. & HER & 1 & 1,6 & & & WD & CF 300 \\
\hline & Inga vera Willd. & TRE & 1 & 1.6 & & & WD & CF 266 \\
\hline & Leucaena leucocephala (Lam.) de Wit & TRE & 1 & 1.6 & & & EX & CF 071 \\
\hline & Macroptilium lathyroides (L.) Urb. & HER & 1 & & & 3.9 & WD & CF 326 \\
\hline & Mimosa debilis Humb. \& Bonpl. ex Willd. & HER & 1 & 1.6 & & & WD & CF 270 \\
\hline & Parapiptadenia rigida (Benth.) Brenan & TRE & 2 & 3.1 & & & $\mathrm{AF}$ & \\
\hline & Rhynchosia edulis Griseb. & CLI & 1 & 1.6 & & & WD & CF 153 \\
\hline & Rhynchosia melanocarpa Grear & CLI & 1 & & & 3.9 & WD & CF 283 \\
\hline & $\begin{array}{l}\text { Senegalia riparia (Kunth) Britton \& Rose ex } \\
\text { Britton \& Killip }\end{array}$ & TRE & 3 & 4.7 & & & WD & \\
\hline & $\begin{array}{l}\text { Senna pendula (Humb. \& Bonpl. ex Willd.) H.S. } \\
\text { Irwin \& Barneby }\end{array}$ & SHR & 1 & & 4.6 & & WD & CF 070 \\
\hline & Sesbania virgata (Cav.) Pers. & TRE & 1 & 1.6 & & & WD & CF 052 \\
\hline & Vigna caracalla (L.) Verdc. & CLI & 1 & 1.6 & & & WD & CF 305 \\
\hline Gentianaceae & Irlbachia alata (Aubl.) Maas & HER & 7 & & 22.7 & 7.7 & WD & CF 284 \\
\hline \multirow[t]{4}{*}{ Lamiaceae } & Aegiphila sellowiana Cham. & TRE & 3 & 4.7 & & & $\mathrm{AF}$ & CF 271 \\
\hline & Hyptis althaeifolia Pohl ex Benth. & HER & 1 & 1.6 & & & WD & CF 331 \\
\hline & Peltodon tomentosus Pohl & HER & 2 & 3.1 & & & WD & CF 064 \\
\hline & Vitex montevidensis Cham. & TRE & 1 & & 4.6 & & $\mathrm{AF}$ & \\
\hline \multirow[t]{6}{*}{ Lauraceae } & Endlicheria paniculata (Spreng.) J. F. Macbr. & TRE & 23 & 28.1 & 13.6 & 7.7 & WD & CF 078 \\
\hline & Nectandra megapotamica (Spreng.) Mez & TRE & 3 & 4.7 & & & $\mathrm{AF}$ & \\
\hline & Nectandra warmingii Meisn. & TRE & 2 & 3.1 & & & $\mathrm{CE}$ & CF 148 \\
\hline & Ocotea lancifolia (Schott) Mez & TRE & 65 & 9.4 & 45.5 & 188.5 & $\mathrm{AF}$ & CF 340 \\
\hline & Ocotea minarum (Nees \& C. Mart.) Mez & TRE & 2 & 3.1 & & & WD & \\
\hline & Persea willdenovii Kosterm. & TRE & 2 & & & 7.7 & $\mathrm{AF}$ & CF 349 \\
\hline Lentibulariaceae & Utricularia gibba $\mathrm{L}$. & HER & 2 & & & 7.7 & WD & \\
\hline \multirow[t]{4}{*}{ Lythraceae } & Cuphea cf. sessiliflora A. St.-Hil. & HER & 1 & 1.6 & & & WD & CF 332 \\
\hline & Cuphea melvilla Lindl. & HER & 1 & & & 3.9 & WD & CF 329 \\
\hline & Cuphea retrorsicapilla Koehne & HER & 1 & 1.6 & & & WD & \\
\hline & Heimia myrtifolia Cham. \& Schltdl. & HER & 1 & 1.6 & & & $\mathrm{AF}$ & CF 330 \\
\hline \multirow[t]{3}{*}{ Malpighiaceae } & Banisteriopsis pubipetala (A. Juss.) Cuatrec. & CLI & 1 & & & 3.9 & WD & CF 190 \\
\hline & Heteropterys sp. & CLI & 1 & & & 3.9 & & CF 293 \\
\hline & Janusia guaranitica (A. St.-Hil.) A. Juss. & CLI & 1 & 1.6 & & & WD & CF 247 \\
\hline \multirow[t]{9}{*}{ Malvaceae } & Ayenia tomentosa $\mathrm{L}$. & HER & 1 & 1.6 & & & $\mathrm{CH}$ & CF 166 \\
\hline & Luehea candicans Mart. & TRE & 8 & 10.9 & 4.6 & & WD & CF 038 \\
\hline & Luehea divaricata Mart. & TRE & 13 & 9.4 & 31.8 & & $\mathrm{AF}$ & CF 047 \\
\hline & Sida cordifolia $\mathrm{L}$. & SHR & 1 & & 4.6 & & WD & CF 264 \\
\hline & Sida linifolia Cav. & HER & 1 & & 4.6 & & WD & CF 309 \\
\hline & Sidastrum paniculatum (L.) Fryxell & HER & 1 & & & & WD & CF 287 \\
\hline & Triumfetta bartramia $\mathrm{L}$. & HER & 35 & 45.3 & 27.3 & & EX & CF 068 \\
\hline & Urena lobata $\mathrm{L}$. & SHR & 1 & 1.6 & & & EX & CF 103 \\
\hline & Wissadula subpeltata (Kuntze) R. E. Fr. & SHR & 1 & 1.6 & & & WD & CF 312 \\
\hline \multirow[t]{2}{*}{ Marantaceae } & Calathea grandiflora K. Schum. & HER & 2 & 3.1 & & & WD & CF 215 \\
\hline & Maranta divaricata Roscoe & HER & 7 & 10.9 & & & WD & CF 211 \\
\hline Mayacaceae & Mayaca sellowiana Kunth & HER & 2 & & & 7.7 & WD & CF 280 \\
\hline \multirow[t]{7}{*}{ Melastomataceae } & Clidemia cf. urceolata DC. & HER & 12 & 1.6 & 27.3 & 19.2 & WD & CF 101 \\
\hline & Clidemia hirta (L.) D. Don & HER & 1 & & 4.6 & & WD & CF 140 \\
\hline & Clidemia $\mathrm{sp}$. & HER & 1 & 1.6 & & & & CF 225 \\
\hline & Miconia chamissois Naudin & SHR & 85 & 3.1 & 45.5 & 280.8 & WD & CF 098 \\
\hline & Miconia theizans (Bonpl.) Cogn. & SHR & 32 & 3.1 & 13.6 & 103.8 & WD & CF 087 \\
\hline & Rhynchanthera verbenoides Cham. & HER & 1 & & & & WD & CF 246 \\
\hline & Rhynchanthera sp. & HER & 7 & & 22.7 & 7.7 & & CF 286 \\
\hline \multirow[t]{2}{*}{ Meliaceae } & Guarea macrophylla Vahl & TRE & 169 & 14.6 & 68.2 & 557.7 & WD & CF 012 \\
\hline & Trichilia elegans A. Juss. & TRE & 10 & 15.6 & & & WD & CF 076 \\
\hline
\end{tabular}


Table 1. Continued.

\begin{tabular}{|c|c|c|c|c|c|c|c|c|}
\hline Family & Species & Habit & $\mathbf{n}$ & NF & SF & SW & $\begin{array}{c}\text { Phyto- } \\
\text { geography }\end{array}$ & $\begin{array}{c}\text { Collection } \\
\text { number }\end{array}$ \\
\hline & Trichilia pallida $\mathrm{Sw}$. & TRE & 1 & 1.6 & & & WD & CF 310 \\
\hline \multirow[t]{2}{*}{ Moraceae } & Ficus cf. luschnathiana (Miq.) Miq. & TRE & 1 & 1.6 & & & $\mathrm{AF}$ & \\
\hline & Ficus sp. & TRE & 1 & & & 3.9 & & \\
\hline Myrsinaceae & Rapanea gardneriana (A. DC.) Mez & TRE & 30 & 6.3 & 27.3 & 76.9 & WD & CF 092 \\
\hline \multirow[t]{17}{*}{ Myrtaceae } & Calyptranthes clusiifolia (Miq.) O. Berg & SHR & 1 & & 4.6 & & $\mathrm{CE}$ & \\
\hline & Calyptranthes lucida Mart. ex DC. & SHR & 16 & 4.7 & & 50.0 & WD & CF 332 \\
\hline & Calyptranthes concinna DC. & SHR & & & & & WD & CF 322 \\
\hline & Calyptranthes sp. & SHR & 8 & 1.6 & & 26.9 & & CF 288 \\
\hline & $\begin{array}{l}\text { Campomanesia guazumifolia (Cambess.) } \\
\text { O. Berg }\end{array}$ & TRE & 1 & 1.6 & & & $\mathrm{AF}$ & CF 191 \\
\hline & Eugenia hiemalis Cambess. & TRE & 1 & 1.6 & & & $\mathrm{AF}$ & CF 150 \\
\hline & Eugenia involucrata DC. & TRE & 1 & 1.6 & & & WD & CF 200 \\
\hline & Eugenia pyriformis Cambess. & TRE & 1 & 1.6 & & & WD & CF 220 \\
\hline & Eugenia speciosa Cambess. & TRE & 1 & 1.6 & & & WD & CF 205 \\
\hline & Eugenia sp.1 & TRE & 1 & & 4.6 & & & \\
\hline & Eugenia sp.2 & TRE & 1 & 1.6 & & & & \\
\hline & Eugenia sp.3 & TRE & 1 & 1.6 & & & & \\
\hline & Myrcia guianensis (Aubl.) DC. & TRE & 4 & 6.3 & & & WD & CF 231 \\
\hline & Myrcia splendens (Sw.) DC. & TRE & 3 & 4.7 & & & WD & CF 224 \\
\hline & Myrcia sp. & TRE & & & & & & CF 255 \\
\hline & Psidium guajava $\mathrm{L}$. & TRE & 2 & 3.1 & & & EX & CF 180 \\
\hline & Indet & TRE & 1 & 1.6 & & & & CF 202 \\
\hline Nyctaginaceae & cf. Guapira & TRE & 1 & 1.6 & & & & CF 176 \\
\hline \multirow[t]{2}{*}{ Ochnaceae } & Ouratea cf. cuspidata Tiegh. & TRE & 2 & 3.1 & & & $\mathrm{AF}$ & CF 253 \\
\hline & Sauvagesia racemosa A. St.-Hil. & HER & 1 & 1.6 & & & WD & CF 106 \\
\hline \multirow[t]{3}{*}{ Onagraceae } & Ludwigia decurrens Walter & HER & 2 & 3.1 & & & WD & CF 268 \\
\hline & Ludwigia sericea (Cambess.) H. Hara & SHR & 21 & 23.4 & 22.7 & 3.9 & WD & CF 093 \\
\hline & Ludwigia tomentosa (Cambess.) H. Hara & SHR & 12 & 1.6 & 18.2 & 26.9 & WD & CF 030 \\
\hline \multirow[t]{10}{*}{ Orchidaceae } & Campylocentrum aromaticum Barb. Rodr. & EPI & 1 & & & 3.9 & $\mathrm{AF}, \mathrm{CE}$ & \\
\hline & Campylocentrum grisebachii Cogn. & EPI & 1 & & & 3.9 & WD & CF 123 \\
\hline & Capanemia micromera Barb. Rodr. & EPI & 1 & & & 3.9 & $\mathrm{AF}$ & \\
\hline & Catasetum sp. & EPI & 1 & 1.6 & & & & \\
\hline & Cochniella jonesiana (Rchb. F). Christenson & EPI & 1 & & & 3.9 & WD & \\
\hline & Habenaria sp. & HER & 1 & & & 3.9 & & CF 353 \\
\hline & $\begin{array}{l}\text { Microlaelia lundii (Rchb. f.) Chiron \& V. P. } \\
\text { Castro }\end{array}$ & EPI & 10 & & & 38.5 & $\mathrm{AF}, \mathrm{CE}$ & CF 021 \\
\hline & Oeceoclades maculata (Lindl.) Lindl. & HER & 14 & 17.2 & 4.6 & 7.7 & WD & CF 051 \\
\hline & Rodriguezia decora (Lem.) Rchb. f. & EPI & 5 & & & 19.2 & $\mathrm{AF}, \mathrm{CE}$ & CF 113 \\
\hline & Sauroglossum nitidum (Vell.) Schltr. & HER & 1 & & & 3.9 & WD & \\
\hline \multirow[t]{2}{*}{ Oxalidaceae } & Oxalis corymbosa DC. & HER & 1 & 1.6 & & & EX & \\
\hline & Oxalis latifolia Kunth & HER & 1 & 1.6 & & & EX & CF 056 \\
\hline \multirow[t]{4}{*}{ Passifloraceae } & Passiflora alata Curtis & CLI & 6 & 6.3 & & 7.7 & WD & CF 074 \\
\hline & Passiflora jilekii Wawra & CLI & 1 & 1.6 & & & $\mathrm{AF}$ & CF 201 \\
\hline & Passiflora misera Kunth & CLI & 1 & 1.6 & & & WD & CF 251 \\
\hline & Passiflora speciosa Gardner & CLI & 1 & 1.6 & & & $\mathrm{AF}$ & CF 133 \\
\hline \multirow[t]{4}{*}{ Piperaceae } & Piper aduncum L. & TRE & 52 & 32.8 & 122.7 & 15.4 & WD & CF 029 \\
\hline & Piper amalago $\mathrm{L}$. & TRE & 3 & 3.1 & & 3.9 & WD & CF 273 \\
\hline & Piper arboreum Aubl. & TRE & 2 & 3.1 & & & WD & CF 058 \\
\hline & Piper umbellatum L. & SHR & 8 & 4.7 & 4.6 & 15.4 & WD & CF 122 \\
\hline \multirow[t]{6}{*}{ Poaceae } & Merostachys sp. & SHR & 1 & 1.6 & & & $\mathrm{AF}$ & $\mathrm{CF} 111$ \\
\hline & Oplismenus hirtellus (L.) P. Beauv. & HER & 4 & 3.1 & & 7.7 & $\mathrm{AF}, \mathrm{CE}$ & \\
\hline & Panicum cyanescens Nees ex Trin. & HER & 1 & & & 3.9 & WD & CF 290 \\
\hline & Panicum sp. & HER & 3 & 4.7 & & & & CF 245 \\
\hline & Pharus lappulaceus Aubl. & HER & 14 & 18.8 & & 7.7 & $\mathrm{AF}$ & CF 135 \\
\hline & Setaria parviflora (Poir.) Kerguélen & HER & 1 & 7.8 & & & WD & CF 272 \\
\hline
\end{tabular}


Table 1. Continued.

\begin{tabular}{|c|c|c|c|c|c|c|c|c|}
\hline Family & Species & Habit & n & NF & SF & SW & $\begin{array}{c}\text { Phyto- } \\
\text { geography }\end{array}$ & $\begin{array}{c}\text { Collection } \\
\text { number }\end{array}$ \\
\hline Rhamnaceae & Gouania mollis Reissek & CLI & 8 & 9.4 & 4.6 & 3.9 & WD & CF 045 \\
\hline Rosaceae & Prunus myrtifolia (L.) Urb. & TRE & 17 & 4.7 & 9.1 & 46.2 & WD & CF 306 \\
\hline \multirow[t]{10}{*}{ Rubiaceae } & Chomelia obtusa Cham. \& Schltdl. & TRE & 8 & 12.5 & & & WD & CF 304 \\
\hline & Coccocypselum lanceolatum (Ruiz \& Pav.) Pers. & HER & 45 & 7.8 & & 153.9 & WD & CF 083 \\
\hline & Emmeorhiza umbellata (Spreng.) K. Schum. & HER & 1 & 1.6 & & & WD & CF 115 \\
\hline & Geophila repens (L.) I. M. Johnst. & HER & 37 & 57.8 & & & WD & CF 062 \\
\hline & Manettia cordifolia Mart. & CLI & 1 & 1.6 & & & WD & CF 315 \\
\hline & Palicourea marcgravii A. St.-Hil. & SHR & 17 & 10.9 & 13.6 & 26.9 & $\mathrm{AF}$ & CF 218 \\
\hline & Psychotria carthagenensis Jacq. & SHR & 31 & 31.3 & & 42.4 & $\mathrm{AF}$ & CF 234 \\
\hline & Psychotria racemosa Rich. & SHR & 18 & 3.1 & 9.1 & 53.9 & WD & CF 079 \\
\hline & Randia nitida (Kunth) DC. & TRE & 1 & 1.6 & & & WD & CF 237 \\
\hline & Indet. & TRE & 18 & 25.0 & 9.1 & & & CF 253 \\
\hline \multirow[t]{3}{*}{ Rutaceae } & $\begin{array}{l}\text { Esenbeckia febrifuga (A. St.-Hil.) A. Juss. ex } \\
\text { Mart. }\end{array}$ & TRE & 7 & 10.9 & & & $\mathrm{AF}, \mathrm{CE}$ & CF 061 \\
\hline & Helietta apiculata Benth. & TRE & 18 & 28.1 & & & $\mathrm{AF}, \mathrm{CE}$ & CF 213 \\
\hline & Zanthoxylum rhoifolium Lam. & TRE & 1 & 1.6 & & & WD & CF 223 \\
\hline Salicaceae & Casearia decandra Jacq. & TRE & 1 & 1.6 & & & WD & CF 221 \\
\hline Santalaceae & Phoradendron piperoides (Kunth) Trel. & PAR & 1 & & & 3.9 & WD & CF 230 \\
\hline \multirow[t]{6}{*}{ Sapindaceae } & $\begin{array}{l}\text { Allophylus edulis (A. St.-Hil., Cambess. \& A. } \\
\text { Juss.) Radlk. }\end{array}$ & TRE & 22 & 31.3 & 9.1 & & WD & CF 165 \\
\hline & Cupania vernalis Cambess. & TRE & 6 & 9.4 & & & $\mathrm{AF}, \mathrm{CE}$ & CF 160 \\
\hline & Matayba elaeagnoides Radlk. & TRE & 9 & 14.6 & & & $\mathrm{AF}, \mathrm{CE}$ & CF 194 \\
\hline & Paullinia pinnata $\mathrm{L}$. & CLI & 2 & 1.6 & & 3.9 & WD & CF 069 \\
\hline & Serjania caracasana (Jacq.) Willd. & CLI & 4 & 1.6 & & 11.5 & WD & CF 131 \\
\hline & Urvillea ulmacea Kunth & CLI & 1 & 1.6 & & & WD & CF 163 \\
\hline Sapotaceae & $\begin{array}{l}\text { Chrysophyllum marginatum (Hook. \& Arn.) } \\
\text { Radlk. }\end{array}$ & TRE & 18 & 21.9 & 4.6 & 11.5 & $\mathrm{AF}, \mathrm{CE}$ & CF 100 \\
\hline \multirow[t]{2}{*}{ Smilacaceae } & Smilax campestris Griseb. & CLI & 16 & 6.3 & 45.5 & 7.7 & WD & CF 084 \\
\hline & Smilax fluminensis Steud. & CLI & 25 & 21.9 & 31.8 & 15.4 & WD & CF 035 \\
\hline \multirow[t]{10}{*}{ Solanaceae } & Capsicum baccatum $\mathrm{L}$. & HER & 1 & & 4.6 & & WD & CF 269 \\
\hline & Capsicum chinense Jacq. & HER & 1 & 1.6 & & & $\mathrm{EX}$ & \\
\hline & Cestrum axillare Vell. & SHR & 9 & 12.5 & 4.6 & & WD & CF 135 \\
\hline & Cestrum mariquitense Kunth & TRE & 1 & 1.6 & & & WD & \\
\hline & Cestrum schlechtendalii G. Don & SHR & 1 & 1.6 & & & WD & \\
\hline & Cestrum strigilatum Ruiz \& Pav. & SHR & 5 & 3.1 & 13.6 & & WD & CF 120 \\
\hline & Physalis angulata $\mathrm{L}$. & HER & 1 & & 4.6 & & WD & CF 189 \\
\hline & Solanum americanum Mill. & HER & 3 & 4.7 & & & WD & CF 053 \\
\hline & Solanum mauritianum Scop. & TRE & 3 & 4.7 & & & $\mathrm{AF}$ & CF 040 \\
\hline & Solanum paniculatum L. & SHR & 1 & 1.6 & & & WD & CF 227 \\
\hline Styracaceae & Styrax pohlii A. DC. & TRE & 47 & 3.1 & 36.4 & 142.4 & WD & CF 239 \\
\hline Theophrastaceae & Clavija nutans (Vell.) B. Stahl & SHR & 7 & & 4.6 & 23.8 & $\mathrm{AF}, \mathrm{CE}$ & CF 162 \\
\hline Thymelaeaceae & Daphnopsis racemosa Griseb. & SHR & 3 & 4.7 & & & $\mathrm{AF}$ & CF 161 \\
\hline \multirow[t]{2}{*}{ Urticaceae } & Cecropia pachystachya Trécul & TRE & 57 & 15.6 & 95.5 & 100.0 & WD & \\
\hline & Boehmeria sp. & HER & 1 & & 4.6 & & & CF 265 \\
\hline Verbenaceae & Lantana camara $\mathrm{L}$. & SHR & 10 & 1.6 & 31.8 & 7.7 & WD & CF 046 \\
\hline Violaceae & Hybanthus communis (A. St.-Hil.) Taub. & HER & 1 & 1.6 & & & WD & \\
\hline Vitaceae & Cissus sp. & CLI & 1 & 1.6 & & & & CF 333 \\
\hline Vochysiaceae & Qualea cordata (Mart.) Spreng. & TRE & 1 & & 4.6 & & $\mathrm{AF}, \mathrm{CE}$ & \\
\hline Xyridaceae & Xyris laxifolia Mart. & HER & 15 & & 22.7 & 38.5 & WD & \\
\hline Zingiberaceae & Hedychium coronarium J. König & HER & 1 & 1.6 & & & EX & \\
\hline
\end{tabular}

pohlii (142.4) presented the highest densities among trees in swampy, and Cecropia pachystachya (95.5) and Guarea macrophylla (68.2) in seasonally flooded habitat.

Density of epiphytes was very low, but they tended to occur in swampy rather than in the other habitats. Six orchid species
(Campylocentrum aromaticum, C. grisebachii, Capanemia micromera, Microlaelia lundii, Cohniella jonesiana and Rodriguezia decora) included 19 individuals exclusively in swampy, and one orchid species (Catasetum sp.) presented one individual in non-flooded habitat (Table 1). The bromeliad 


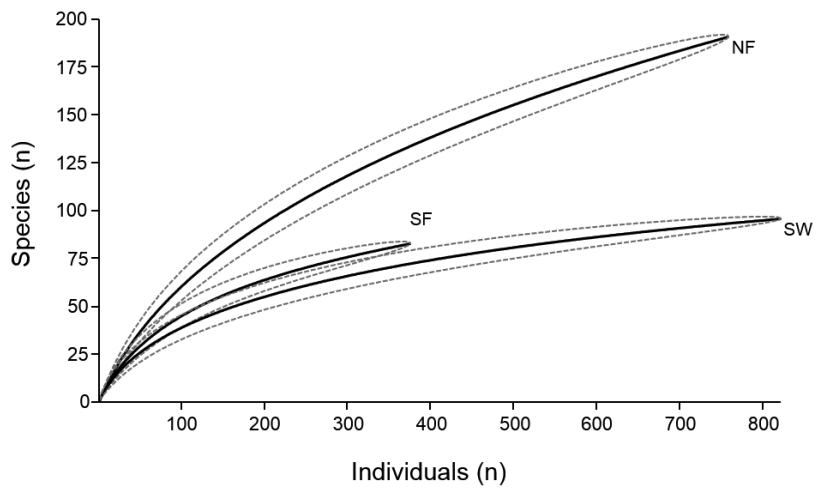

Figure 2. Rarefaction curves for cumulative species according to number of reproductive individuals sampled in non-flooded (NF), seasonally flooded (SF) and swampy (SW) habitats in the riparian forests of Touro and Tarumã streams, southwestern Brazil. Dotted lines show $95 \%$ Confidence Intervals.

Figura 2. Curvas de rarefação de espécies com base na quantidade de indivíduos reprodutivos amostrados em habitat não inundável (NF), sazonalmente inundável (SF) e permanentemente inundado (SW) nas florestas ripárias dos rios Touro e Tarumã, sudoeste do Brasil. Linhas pontilhadas mostram Intervalos de Confiança de $95 \%$

Aechmea bromeliifolia showed one individual in swampy and another in non-flooded habitat, where also one individual of the epiphytic cactus Epiphyllum phyllanthus occurred.

Based on proportional distribution of species with sample size $\geq 10$ individuals, most of them presented higher abundance in swampy (25) than in seasonally (10) or non-flooded habitats (14). This pattern was also consistent within habits - herbs, shrubs or trees (Figure 3). The most frequent species in swampy were Coccocypselum lanceolatum, Cyperus haspan, Calyptrocarya glomerulata, Scleria melaleuca, Begonia cucullata, Xyris laxifolia, Scleria bancana, Smilax campestris (herbs), Gaylussacia brasiliensis, Miconia chamissois, M. theizans, Psychotria racemosa, Ludwigia tomentosa (shrubs), Geonoma brevispatha, Calyptranthes lucida, Guarea macrophylla, Styrax pohlii, Ocotea lancifolia, Ilex brasiliensis, Prunus myrtifolia, Rapanea gardneriana, Tapirira guianensis, Cecropia pachystachya (trees). On the other hand, in non-flooded habitat were Geophila repens, Rhynchospora marisculus, Elephantopus mollis, Triumfetta bartramia, Pharus lappulaceus, Oeceoclades maculata (herbs), Ludwigia sericea, Chrysophyllum marginatum (shrubs), Helietta apiculata, Trichilia elegans, Gochnatia polymorpha, Allophylus edulis, Endlicheria paniculata, and Sebastiania brasiliensis (trees). Common species in seasonally flooded habitat were Costus arabicus, Canna indica, Smilax fluminensis, Chromolaena maximilianii, Conyza bonariensis (herbs), Piper aduncum, Lantana camara, Clidemia cf. urceolata (shrubs), Luehea divaricata and Syagrus romanzoffiana (trees). The pioneer tree Cecropia pachystachya was equally abundant in swampy and seasonally flooded habitats (Figure 3).

\section{Discussion}

The flora we inventoried seems to be markedly similar to other Atlantic Forest floras elsewhere, as all recorded species indeed occur throughout this domain (sensu Ab'Saber 2000), including two regarded as endemic, Capanemia micromera (Orchidaceae) and Passiflora jilekii (Passifloraceae) (Cervi 1997, Stehmann et al. 2009, Buzatto \& Machado 2011). The richest families in our study site (Asteraceae, Fabaceae, Myrtaceae, Cyperaceae, Malvaceae,

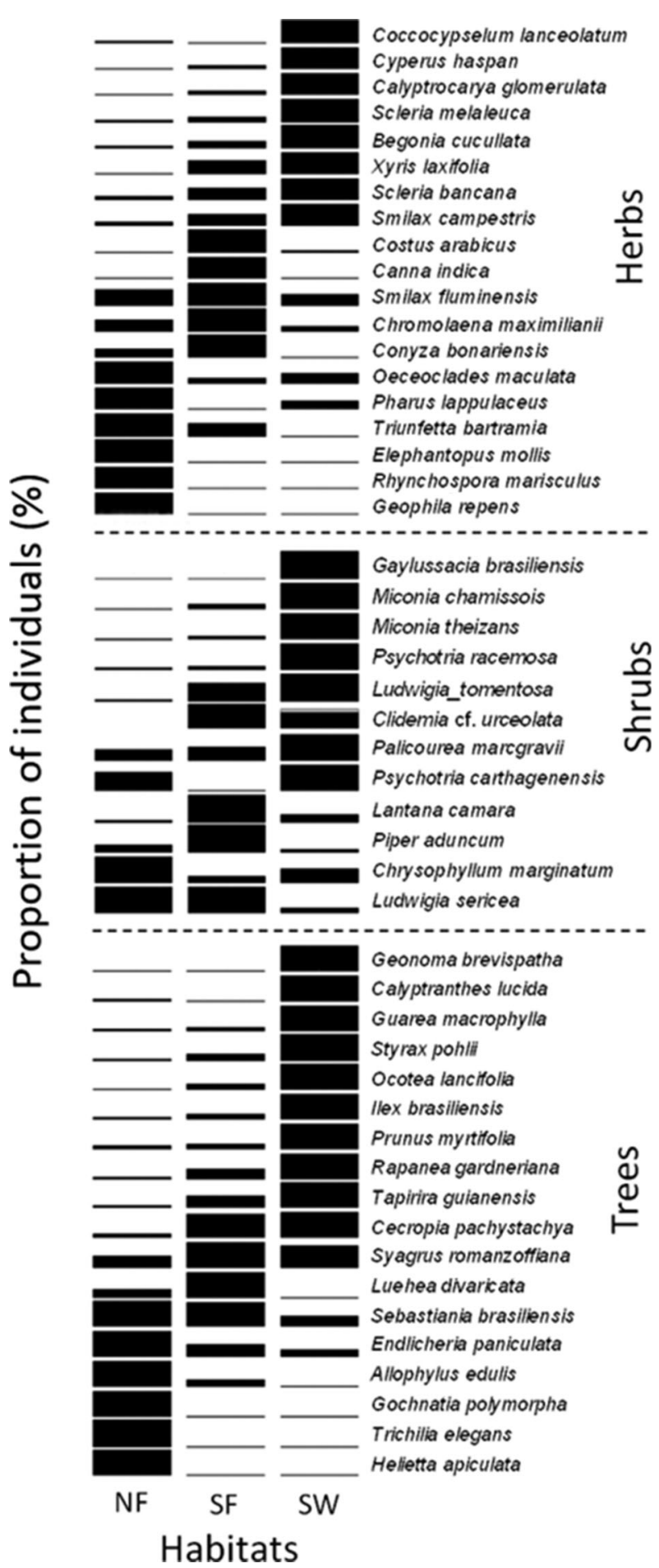

Figure 3. Commonest herbaceous, shrub and tree species $(\geq 10$ individuals) ordinated according to non-flooded (NF), seasonally flooded (SF) and swampy (SW) habitats along Touro and Tarumã riparian forests, Mato Grosso do Sul. Black bars indicate the proportion $(\%)$ of reproductive individuals per habitat for each species, estimated for areas of equal sizes among habitats.

Figura 3. Espécies herbáceas, arbustivas e arbóreas mais comuns ( $>10$ indivíduos) ordenadas conforme os tipos de habitats, não inundável (NF), sazonalmente inundável (SF) e permanentemente inundado (SW), ao longo da floresta ripária do Touro e Tarumã, Mato Grosso do Sul. Barras pretas indicam a proporção (\%) de indivíduos reprodutivos por habitat para cada espécie, estimada para áreas de tamanhos iguais entre os habitats. 
Orchidaceae, Rubiaceae and Solanaceae) also show high number of species in other Atlantic Forest sites (Romagnolo \& Souza 2000, Rodrigues \& Nave 2004, Stehmann et al. 2009). Furthermore, the local high representativeness of Orchidaceae and low richness of some families, such as Moraceae, differ from the expected for riparian forests in the state's Cerrado domain (Pott et al. 2006, Lehn et al. 2008, Baptista-Maria et al. 2009). Therefore, flora of Touro and Tarumã riparian forests add support to sustain the southeastern Mato Grosso do Sul as an inland region of the Atlantic Forest domain, as formerly suggested by Rizzini (1979).

We found several species that have not been reported in the Mato Grosso do Sul regions of Cerrado or Pantanal, which reinforces the influence of the Atlantic Forest domain on our study site. The orchid Campylocentrum grisebachii is known to occur in the Atlantic Forest and Amazon, and our record increases its known distribution (Johnson 2001, Barros et al. 2010) towards the center of South America. In addition, the distribution of Capanemia micromera is known along Atlantic Forest in Brazil, and in Paraguay, Uruguay and Argentina (Johnson 2001, Barros et al. 2010, Buzatto \& Singer 2010, Buzatto et al. 2011), thus our record enlarges its distribution to $290 \mathrm{~km}$ northward and $150 \mathrm{~km}$ eastward. Our record of Microlaelia lundii (Orchidaceae), distributed in the Atlantic Forest and Cerrado domains (Barros et al. 2010), increases the species' distribution to $290 \mathrm{~km}$ north, $650 \mathrm{~km}$ south (Dubs 1998), and $300 \mathrm{~km}$ westward (Barros et al. 2010). Other orchid species recorded here for the first time in Mato Grosso do Sul have been found 100 and $300 \mathrm{~km}$ apart, respectively Rodriguezia decora in Atlantic Forest and Cerrado (Johnson 2001, Souza et al, 2009, Barros et al. 2010) and Sauroglossum nitidum in Atlantic Forest, Cerrado and Caatinga (Barros et al. 2010). The occurrence of Passiflora jilekii in the Touro and Tarumã riparian forests increases its distribution $1100 \mathrm{~km}$ westward (Forzza et al. 2010, Cervi et al. 2010). For Persea willdenovii (Lauraceae), our record is $600 \mathrm{~km}$ northwestern from its previous known distribution (Quinet et al. 2010, Forzza et al. 2010). Additional species recorded for the first time in Mato Grosso do Sul Merremia macrocalyx (Convolvulaceae), Apteria aphylla (Burmanniaceae) and Qualea cordata (Vochysiaceae) - might be expected since they are widely distributed throughout different domains (Dubs 1998, Bianchini \& Ferreira 2010, Forzza et al. 2010, França 2010, Maas \& Maas 2010). Ilex brasiliensis (Aquifoliaceae), Gaylussacia brasiliensis (Ericaceae), Daphnopsis racemosa (Thymelaeaceae), Condylocarpon isthmicum (Apocynaceae) and Citronella gongonha (Cardiopteridaceae) were previously recorded in mid-east regions of Cerrado in Mato Grosso do Sul only (Pott et al. 2006).

In general, the studied flora is highly composed of herbs and contains few tree species, compared to other riparian forests in the Atlantic Forest domain or even elsewhere in Brazil (Oliveira-Filho \& Ratter 1995, Metzger et al. 1997, Oliveira-Filho \& Fontes 2000, Felfili et al. 2001, Souza et al. 2009). The extension of wetter soils - swampy and seasonally flooded - could partially explain such differences, as they are highly occupied by herbs at the study site. Tree richness is otherwise expected to be favored in non-flooded habitats, where we recorded several species with low abundances. On the other hand, the most abundant species within any habit are the flood-tolerant ones, which occur in the habitats with reduced number of species. This pattern agrees with the expectation that non-flooded sites allow occurrence of most plant species, whose abundances are mainly regulated by interspecific competition, whereas environmental filter determines plant species under flood conditions (Ivanauskas et al. 1997, Fischer \& Santos 2001, Marques et al. 2003, Lobo \& Joly 2004).

Richness of epiphytes in Touro and Tarumã forests is lower than that in other Atlantic Forest regions with less seasonality and higher annual rainfall than in our study site $(1600 \mathrm{~mm})$, as indeed expected since humidity has been regarded as a main factor affecting epiphytic growth (Fischer \& Araujo 1995, Rogalski \& Zanin 2003, Bataghin et al. 2012; Forzza et al. 2014). Likewise, epiphytes were richer and more abundant in swampy than in non-flooded habitat at the study site probably due to higher humidity in the former. However, additional factors are likely contributing to low richness of epiphytes at the study site, since other Atlantic Forest sites with similar annual rainfall can present remarkably more epiphytic species (Forzza et al. 2014). The narrow forest remnants along Touro and Tarumã streams are enclosed in pasture and cultivated landscapes, thus different sources of disturbance can contribute to the low richness of epiphytes at the study site (Wolf 1994, Pinto et al. 1995, Barthlot et al. 2001, Rogalski \& Zanin 2003).

Overall, flora of Touro and Tarumã riparian forests contains several components expected for the Atlantic Forest, although it predominantly included species widespread in different domains. The local flood gradient may favor plant richness. A certain level of disturbance is indeed apparent at the study site due to the relatively high abundance of herbs, the lack of epiphytes in some suitable sites, the conspicuous presence of weedy pioneers, and the occurrence of exotic invaders. The new occurrences for Mato Grosso do Sul and the new limits of distribution for plant species surveyed here highlight the importance of conservation of the remaining forests in the westernmost Atlantic Forest.

\section{Acknowledgements}

To Geraldo A. Damasceno-Júnior, Vali J. Pott, Ângela L. B. Sartori, Marcos Sobral, Flávio M. Alves and Glaucia de A. Moraes for help with species identification; and to Grupo de Estudos em Proteção à Biodiversidade (GEBIO), Public Ministry of Naviraí, and JBS cattle abattoir for supporting the project. Claudenice Faxina was supported by CAPES grant, Erich Fischer by CNPq grant, and Arnildo Pott by CAPES and CNPq grants.

\section{References}

AB'SABER, A.N. 2000. The natural organization of Brazilian inter- and subtropical landscapes. Revista do Instituto Geológico 21: $57-70$.

APG III. 2009. An update of the Angiosperm Phylogeny Group classification for the orders and families of flowering plants: APG III. Bot. J. Linn. Soc. 161:105-121, http://dx.doi.org/10.1111/ (ISSN) 1095-8339

ARRUDA, L. \& DANIEL, O. 2007. Florística e diversidade em um fragmento de floresta estacional semidecidual aluvial em Dourados-MS. Floresta 37(2):189-199.

BAPTISTA-MARIA, V.R., RODRIGUES, R.R., DAMASCENOJUNIOR, G.A., SOUZA MARIA, F. \& SOUZA, V.C. 2009. Composição florística de florestas estacionais ribeirinhas no estado de Mato Grosso do Sul, Brasil. Acta Bot. Bras. 23:535-548, http://dx.doi.org/10.1590/S0102-33062009000200025

BARTHLOT, W., SCHMIT-NEUERBURG, V., NIEDER, J. \& ENGWALD, S. 2001. Diversity and abundance of vascular epiphytes: a composition of secondary vegetation and primary montane rain forest in the Venezuelan Andes. Plant Ecol. 152:145-156, http://dx.doi.org/10.1023/A:1011483901452 
BARROS, F.D.E., VINHOS, F., RODRIGUES, V.T., BARBERENA, F.F.V.A. \& FRAGA, C.N. 2010. Orchidaceae In Lista de espécies da flora do Brasil. Jardim Botânico do Rio de Janeiro. (http://floradobrasil.jbrj.gov.br/2010/FB011274).

BATAGHIN, F.A., MULLER, A., PIRES, J.S.R., BARROS, F., FUSHITA, A.T. \& SCARIOT, E.C. 2012. Riqueza e estratificação vertical de epífitas vasculares na Estação Ecológica de Jataí - área de Cerrado no sudeste do Brasil. Hoehnea 39(4):615-626.

BIANCHINI, R.S. \& FERREIRA, P.P.A.2010. Convolvulaceae In Lista de espécies da flora do Brasil. Jardim Botânico do Rio de Janeiro. (http://floradobrasil.jbrj.gov.br/2010/FB007100).

BUZATTO, C R. \& SINGER, R B. 2010. O gênero Capanemia Barb. Rodr. (Oncidiinae: Orchidaceae). Rev. Bras. Biociênc. 8:309-323.

BUZATTO, C R. \& MACHADO, M C. 2011. Capanemia Barb. Rodr. (Orchidaceae: Oncidiinae), a new record from Bahia state, Brazil. Acta Bot. Bras. 25:249-251.

BUZATTO, C R., SINGER, R B. \& ROMERO-GONZALEZ, G A. 2011. Typifications and new synonymies in Capanemia (Orchidaceae, Oncidiinae). Novon 21:28-33.

CERVI, A.C. 1997. Passifloraceae do Brasil: estudo do gênero Passiflora L. subgênero Passiflora. Fontqueria 45:1-92.

CERVI, A.C., MILWARD-DE-AZEVEDO, M.A. \& BERNACCI, L. C. 2010. Passifloraceae In Lista de espécies da flora do Brasil. Jardim Botânico do Rio de Janeiro. (http://floradobrasil.jbrj.gov. br/2010/FB012537).

DAMASCENO-JUNIOR, G.A., POTT, A., POTT, V.J. \& SILVA, J. S.V. 2009. Florestas estacionais no Pantanal, considerações florísticas e subsídios para conservação. Geografia 34: 697-707.

DUBS, B. 1998. Prodromus florae matogrossensis. Küssnacht, Betrona Verlag.

FELFILI, J.M., MENDONÇA, R.C., WALTER, B.M.T., SILVA JUNIOR, M.C., NÓBREGA, M.G.G., FAGG, C.W., SEVILHA, A.C. \& SILVA, M.A. 2001. Flora fanerogâmica das matas de galeria e ciliares do Brasil Central. In Cerrado caracterização e recuperação de matas de galeria (J. F. Ribeiro, C. E. L. da Fonseca \& J. C. SousaSilva, eds.). Embrapa Cerrados, Planaltina, DF, Brasil. p.195-263.

FISCHER, E.A. \& ARAUJO, A.C. 1995. Spatial organization of a bromeliad community in the Atlantic rainforest, south-eastern Brazil. J. Trop. Ecol. 11: 559-567, http://dx.doi.org/10.1017/S0266467400009123

FISCHER, E. \& SANTOS, F.A.M. 2001. Demography, phenology and sex of Calophyllum brasiliense (Clusiaceae) trees in the Atlantic Forest. J. Trop. Ecol. 17: 903-909, http://dx.doi.org/10.1017/S0266467401001675

FORZZA, R.C., LEITMAN, P.M., COSTA, A.F., CARVALHO JR., A.A, PEIXOTO, A.L, WALTER, B.M.T, BICUDO, C, ZAPPI, D, COSTA, D.P, LLERAS, E, MARTINELLI, G, LIMA, H.C, PRADO, J, STEHMANN, J.R, BAUMGRATZ, J.F.A, PIRANI, J.R, SYLVESTRE, L, MAIA, L.C, LOHMANN, L.G, QUEIROZ, L.P, SILVEIRA, M, COELHO, M.N, MAMEDE, M.C, BASTOS, M.N.C, MORIM, M.P, BARBOSA, M.R, MENEZES, M, HOPKINS, M, SECCO, R, CAVALCANTI, T.B \& SOUZA, V.C. 2010. Catálogo de plantas e fungos do Brasil. Andréa Jakobsson Estúdio, Jardim Botânico do Rio de Janeiro, Rio de Janeiro, v.2.

FORZZA, R.C., PIFANO, D.S., OLIVEIRA-FILHO, A.T., MEIRELES, L.D., FARIA, P.L., SALIMENA, F.R., MYNSSEN, C.M. \& PRADO, J. 2014. Flora vascular da Reserva Biológica da Represa do Grama, Minas Gerais, e sua relação florística com outras florestas do sudeste brasileiro. Rodriguésia 65: 275-292.

FRANÇA, F. 2010. Vochysiaceae In Lista de espécies da flora do Brasil. Jardim Botânico do Rio de Janeiro. (http://floradobrasil. jbrj.gov.br/2010/FB015282).

GUEDES-BRUNI, R.R., MORIM, M.P.M., LIMA, H.C. \& SYLVESTRE, L.S., 2002. Inventário florístico. In Manual metodológico para estudos botânicos na Mata Atlântica (L.S., Sylvestre \& M.M.T., Rosa, eds.). Rio de Janeiro. p.24-49.

HAMMER, Ø., HARPER, D.A.T. \& RYAN, P.D. 2001. PAST: Paleontological statistics software package for education and data analysis. Palaeontologia Electronica 4(1): 9pp. http://palaeo-electronica. org/2001_1/past/issue1_01.htm
IBGE. 1984. Naviraí - Centro-Oeste - Mato Grosso do Sul. Coleção de monografias municipais. N. 101, Rio de Janeiro-RJ.

IVANAUSKAS, N.M., RODRIGUES, R.R. \& NAVE, A G. 1997. Aspectos ecológicos de um trecho de floresta de brejo em Itatinga, SP: florística, fitossociologia e seletividade de espécies. Rev. Bras. Bot. 20:139-153, http://dx.doi.org/10.1590/S0100-84041997000200005

JOHNSON, A.E. 2001. Las Orquídeas del Parque Nacional Iguazú. LOLA, Buenos Aires, Argentina.

LEHN, C.R., ALVES, F.M. \& DAMASCENO-JUNIOR, G.A. 2008. Fitossociologia de um cerrado sensu-stricto na região da borda oeste do Pantanal, Corumbá, MS. Pesquisa Botânica 59: 129-142.

LOBO, P.C. \& JOLY, C.A. 2004. Aspectos ecofisiológicos da vegetação de mata ciliar de mata ciliar do sudeste do Brasil. In Matas ciliares: conservação e recuperação (R. R. Rodrigues \& H. F. Leitão-Filho,eds.). São Paulo. Edusp/Fapesp, p.143-157.

MAAS, P. \& MAAS, H. 2010. Burmanniaceae In Lista de espécies da flora do Brasil. Jardim Botânico do Rio de Janeiro. (http:// floradobrasil.jbrj.gov.br/2010/FB110590).

MARQUES, M.C.M., SILVA, S.M. \& SALINO, A. 2003. Florística e estrutura do componente arbustivo-arbóreo de uma floresta higrófila da bacia do rio Jacaré-Pepira, SP, Brasil. Acta Bot. Bras. 17:495-506, http://dx.doi.org/10.1590/S0102-33062003000400002

METZGER, J.P., BERNACCI, L.C. \& GOLDENBERG, R. 1997. Pattern of tree species diversity in riparian forest fragments with different widths (SE Brazil). Plant Ecol. 133:135-152, http://dx.doi. org/10.1023/A:1009791831294

NOGUCHI, D.K., PETINE, G.N. \& SARTORI, A.L.B. 2009. Florística e síndromes de dispersão de espécies arbóreas em remanescentes de Chaco de Porto Murtinho, Mato Grosso do Sul. Rodriguésia 60:353-365.

OLIVEIRA-FILHO, A.T. \& RATTER, J.A. 1995. A study of the origin of central Brazilian forests by the analysis of plant species distribution patterns. Edinburgh J. Bot. 52:141-194, http://dx.doi. org/10.1017/S0960428600000949

OLIVEIRA-FILHO, A.T. \& FONTES, M.A.L. 2000. Patterns of floristic differentiation among Atlantic forests in southeastern Brazil and the influence of climate. Biotropica 32:793-810.

PINTO, A.C.R., DEMATTÊ, M.E.S.P. \& PAVANI, M.C.M.D. 1995. Composição florística de epífitas (Magnoliophyta) em fragmento de floresta no município de Jaboticabal, SP, Brasil. Científica 23: 283-289.

POTT, A. \& POTT, V.J. 1994. Plantas do Pantanal. Embrapa, Brasília. POTT, V.J. \& POTT, A. 2000. Plantas aquáticas do Pantanal. Embrapa, Brasília.

POTT, A. \& POTT, V.J. 2003. Espécies de fragmentos florestais em Mato Grosso do Sul. In Fragmentação florestal e alternativas de desenvolvimento rural na região Centro-Oeste (Costa, R. B., ed.). UCDB. Campo Grande, p.26-52.

POTT, A., POTT, V.J., SCIAMARELLI, A., SARTORI Â.L.B., SCREMIN-DIAS, E., JACQUES, E. de L., ARAGAKI, S., NAKAJIMA, J.N., ROMERO R., CRISTALDO, A.C. de M. \& DAMASCENO-JUNIOR, G.A. 2006. Flora - Inventário das Angiospermas no Complexo Aporé-Sucuriu In Biodiversidade do Complexo Aporé-Sucuriu - Subsídios à conservação e manejo do cerrado - Área prioritária 316 (Pagotto, T. C. S. \& Souza, P. R. de, eds.).UFMS, Campo Grande, p. 45-66.

QUINET, A., BAITELLO, J.B. \& MORAES, P.L.R DE 2010. Lauraceae In Lista de espécies da flora do Brasil. Jardim Botânico do Rio de Janeiro. (http://floradobrasil.jbrj.gov.br/2010/FB008523).

R DEVELOPMENT CORE TEAM 2010. R: A language and environment for statistical computing. Vienna: R Foundation for Statistical Computing (http://www.R-project.org).

RIZZINI, C T. 1979. Tratado de fitogeografia do Brasil. v.2. Aspectos ecológicos. Hucitec-Edusp, São Paulo.

RODRIGUES, R.R., NAVE A.G., 2004. Heterogeneidade florística das matas ciliares In Matas ciliares: conservação e recuperação (R. R. Rodrigues \& H. F. Leitão-Filho, eds.). São Paulo. Edusp/ Fapesp, p.45-71. 
Faxina, C. et al.

RODRIGUES, R.R. \& SHEPHERD, G.J. 2004. Fatores condicionantes da vegetação ciliar In Matas ciliares: conservação e recuperação (R. R. Rodrigues \& H. F. Leitão-Filho, eds.). São Paulo. Edusp/Fapesp, p.101-107.

ROGALSKI, J.M. \& ZANIN, E.M. 2003. Composição florística de epífitos vasculares no estreito de Augusto César, floresta estacional decidual do alto Uruguai, RS, Brasil. Rev. Bras. Bot. 26:551-556, http://dx.doi.org/10.1590/S0100-84042003000400014

ROMAGNOLO, M.B. \& SOUZA, M.C. 2000. Analise florística e estrutural de florestas ripárias do alto rio Paraná, Taquaruçu, MS. Acta Bot. Bras. 14:163-174.

SALIS, S.M., SILVA, M.P., MATTOS, P.P., SILVA, J.S.V., POTT, V. J. \& POTT, A. 2004. Fitossociologia de remanescentes de floresta estacional decidual em Corumbá, estado de Mato Grosso do Sul,
Brasil. Rev. Bras. Bot. 27: 671-684, http://dx.doi.org/10.1590/ S0100-84042004000400008

SOUZA, M.C., KAWAKITA, K., SLUSARSKI, S.R. \& PEREIRA, G.F. 2009. Vascular flora of the Upper Paraná River floodplain. Braz. J. Biol. 69: 735-745, http://dx.doi.org/10.1590/S1519-69842009000300027 STEHMANN, J.R., FORZZA, R.C., SALINO, A., SOBRAL, M., COSTA, D.P. \& KAMINO, L.H.Y. 2009. Plantas da Floresta Atlântica. Jardim Botânico do Rio de Janeiro, Rio de Janeiro.

VELOSO, H.P., RANGEL FILHO, A.L. \& LIMA, J.C.A. 1991. Classificação da vegetação brasileira, adaptada a um sistema universal. IBGE, Rio de Janeiro.

WOLF, J.H.D. 1994. Factors controlling the distribution of vascular and nonvascular epiphytes in the northern Andes. Vegetatio 112:15-28. 